International Journal of Pure and Applied Mathematics

Volume 86 No. 4 2013, 701-720

ISSN: 1311-8080 (printed version); ISSN: 1314-3395 (on-line version)

url: http://www.ijpam.eu

doi: http://dx.doi.org/10.12732/ijpam.v86i4.9

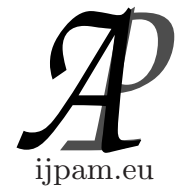

\title{
EXISTENCE OF SOLUTIONS FOR NONLINEAR SECOND-ORDER $Q$-DIFFERENCE EQUATIONS WITH THREE-POINT MULTI-TERM $q$-INTEGRAL BOUNDARY CONDITIONS
}

\author{
Jessada Tariboon $^{1}$, Weerawat Sudsutad ${ }^{2} \S$ \\ ${ }^{1,2}$ Department of Mathematics \\ Faculty of Applied Science \\ King Mongkut's University of Technology \\ North Bangkok, Bangkok, 10800, THAILAND
}

\begin{abstract}
In this paper is concerned with the existence of solutions for nonlinear second-order $q$-difference equations with three-point multi-term $q$ integral boundary conditions. Some new existence results are obtained by using Banach's contraction mapping, Krasnoselskii's fixed point theorem and LeraySchauder degree theory. As an application, we give two examples that illustrate our results.
\end{abstract}

AMS Subject Classification: 34B10, 39A13

Key Words: existence, $q$-difference equation, $q$-derivative, $q$-integral, boundary value problem

\section{Introduction}

The study of $q$-calculus was initiated in the beginning of the twentieth century by the pioneer works of Jackson [1], Carmichael [2], Mason [3], Adams [4], Trjitzinsky [5], etc. In the last few decades, the $q$-theory has involved into a variety of discipline areas of research not only in mathematics but also covering other fields of science and its applications as well. See [6-14]. For some recent

Received: April 13, 2013

(c) 2013 Academic Publications, Ltd.

$\S$ Correspondence author url: www.acadpubl.eu 
work, we refer the reader to [15-26] and references therein. However, there are only a few papers that have studied the existence of solutions for boundary value problems of nonlinear $q$-difference equations, see [27-29].

In [30], Ahmad studied the existence of solutions for nonlinear third-order $q$-difference equation with boundary conditions

$$
\begin{gathered}
D_{q}^{3} u(t)=f(t, u(t)), \quad 0 \leq t \leq 1, \\
u(0)=0, \quad D_{q} u(0)=0, \quad u(1)=0 .
\end{gathered}
$$

He obtained some existence results by using standard fixed point theorems and Leray-Schauder degree theory. He also showed that if $q \rightarrow 1$ then his results corresponded to the classical results. In [31], Ahmad, Alsaedi and Ntouyas established the existence criteria for nonlinear second-order $q$-difference equation with non-separated boundary conditions

$$
\begin{gathered}
D_{q}^{2} u(t)=f(t, u(t)), \quad t \in[0, T], \\
u(0)=\eta u(T), \quad D_{q} u(0)=\eta D_{q} u(T) .
\end{gathered}
$$

In [32], Pongam, Asawasumrit, Tariboon investigated the sequential derivatives of the nonlinear $q$-difference equation with three-point $q$-integral boundary condition of the form

$$
\begin{gathered}
D_{q}\left(D_{p}+\lambda\right) u(t)=f(t, u(t)), \quad t \in[0, T], \\
u(0)=0, \quad \beta \int_{0}^{\eta} u(s) d_{r} s=u(T), \quad \eta \in(0, T) .
\end{gathered}
$$

Using fixed point theorems and Leray-Schauder degree theory, some existence were obtained.

In this paper, we consider the following nonlinear second-order $q$-difference equation with three-point multi-term $q$-integral boundary conditions

$$
\begin{gathered}
D_{q}^{2} u(t)=f(t, u(t)), \quad 0 \leq t \leq T, \\
u(0)=\sum_{i=1}^{n} \alpha_{i} \int_{0}^{\eta} u(s) d_{p_{i}} s, \quad u(T)=\sum_{j=1}^{m} \beta_{j} \int_{0}^{\eta} u(s) d_{r_{j}} s,
\end{gathered}
$$

where $0<q, p_{i}, r_{j}<1, i=1,2, \ldots, n, j=1,2, \ldots, m$ and $\alpha_{i}, \beta_{j} \in R$ are such that $\eta^{2}\left(\sum_{j=1}^{m} \frac{\beta_{j}}{1+r_{j}}-\sum_{i=1}^{n} \frac{\alpha_{i}\left(1-\eta \sum_{j=1}^{m} \beta_{j}\right)}{1+p_{i}}\right) \neq T, \eta \in(0, T)$ is a fixed constant. We note that the boundary conditions (2) have different values of the $q$-numbers of $q$-integral at the boundary points. The existence results of problem (1)-(2) have not been established, previously. 


\section{Preliminaries}

In this section, we introduce notation, some basic concepts of $q$-calculus and prove a lemma before starting our main results.

For $0<q<1$, we define the $q$-derivative of a real valued function $f$ as

$$
D_{q} f(t):=\frac{f(t)-f(q t)}{(1-q) t},
$$

and $D_{q} f(0)=\lim _{t \rightarrow 0} D_{q} f(t)$ provided $f^{\prime}(0)$ exists. The higher order $q$-derivatives are given by

$$
D_{q}^{0} f(t)=f(t), \quad D_{q}^{n} f(t)=D_{q} D_{q}^{n-1} f(t), \quad n \in N .
$$

The $q$-integral of a function $f$ defined in the interval $[a, b]$ is given by

$$
\int_{a}^{t} f(s) d_{q} s:=\sum_{n=0}^{\infty}(1-q) q^{n}\left[t f\left(t q^{n}\right)-a f\left(q^{n} a\right)\right], \quad t \in[a, b] .
$$

For $a=0$, we denote

$$
I_{q} f(t)=\int_{0}^{t} f(s) d_{q} s=\sum_{n=0}^{\infty} t(1-q) q^{n} f\left(t q^{n}\right),
$$

provided the series converges. If $a \in[0, b]$ and $f$ is defined in the interval $[0, b]$, then

$$
\int_{a}^{b} f(t) d_{q} t=\int_{0}^{b} f(t) d_{q} t-\int_{0}^{a} f(t) d_{q} t
$$

Note that

$$
D_{q} I_{q} f(t)=f(t)
$$

and if $f$ is continuous at $t=0$, then

$$
I_{q} D_{q} f(t)=f(t)-f(0) .
$$

In $q$-calculus, the product rule and integration by parts formula are

$$
\begin{aligned}
D_{q}(g h)(t) & =\left(D_{q} g(t)\right) h(t)+q(q t) D_{q} h(t) \\
\int_{0}^{t} h(s) D_{q} g(s) d_{q} s & =[h(s) g(s)]_{0}^{t}-\int_{0}^{t} D_{q} h(s) g(q s) d_{q} s .
\end{aligned}
$$

Further, reversing the order of integration is given by

$$
\int_{0}^{t} \int_{0}^{s} f(r) d_{q} r d_{q} s=\int_{0}^{t} \int_{q r}^{t} f(r) d_{q} s d_{q} r .
$$

In the limit $q \rightarrow 1$ the $q$-calculus corresponds to the classical calculus. 
Lemma 1. Let $\eta^{2}\left(\sum_{j=1}^{m} \frac{\beta_{j}}{1+r_{j}}-\sum_{i=1}^{n} \frac{\alpha_{i}\left(1-\eta \sum_{j=1}^{m} \beta_{j}\right)}{1+p_{i}}\right) \neq T$ and $0<$ $q, p_{i}, r_{j}<1$ for $i=1,2, \ldots, n, j=1,2, \ldots, m$. Then for $h \in C[0, T]$, the boundary value problem

$$
\begin{gathered}
D_{q}^{2} u(t)=h(t), \quad 0<t<T \\
u(0)=\sum_{i=1}^{n} \alpha_{i} \int_{0}^{\eta} u(s) d_{p_{i}} s, \quad u(T)=\sum_{j=1}^{m} \beta_{j} \int_{0}^{\eta} u(s) d_{r_{j}} s,
\end{gathered}
$$

has the unique solution

$$
\begin{aligned}
u(t)= & \int_{0}^{t}(t-q s) h(s) d_{q} s \\
+ & {\left[\left(1-\eta \sum_{i=1}^{n} \alpha_{i}\right) t+\eta^{2} \sum_{i=1}^{n} \frac{\alpha_{i}}{1+p_{i}}\right] \int_{0}^{T} \frac{(T-q s)}{\Phi} h(s) d_{q} s } \\
- & {\left[\left(1-\eta \sum_{i=1}^{n} \alpha_{i}\right) t+\eta^{2} \sum_{i=1}^{n} \frac{\alpha_{i}}{1+p_{i}}\right] } \\
& \times \sum_{j=1}^{m} \frac{\beta_{j}}{\Phi} \int_{0}^{\eta} \int_{0}^{s}(s-q \nu) h(\nu) d_{q} \nu d_{r_{j}} s \\
& +\left[\left(1-\eta \sum_{j=1}^{m} \beta_{j}\right) t-T+\eta^{2} \sum_{j=1}^{m} \frac{\beta_{j}}{1+r_{j}}\right] \\
& \quad \times \sum_{i=1}^{n} \frac{\alpha_{i}}{\Phi} \int_{0}^{\eta} \int_{0}^{s}(s-q \nu) h(\nu) d_{q} \nu d_{p_{i}} s,
\end{aligned}
$$

where

$$
\Phi=\left(1-\eta \sum_{i=1}^{n} \alpha_{i}\right)\left(\eta^{2} \sum_{j=1}^{m} \frac{\beta_{j}}{1+r_{j}}-T\right)-\eta^{2} \sum_{i=1}^{n} \frac{\alpha_{i}\left(1-\eta \sum_{j=1}^{m} \beta_{j}\right)}{1+p_{i}} .
$$

Proof. For $t \in[0, T]$, we get by $q$-integrating for (6) that,

$$
D_{q} u(t)=\int_{0}^{t} h(s) d_{q} s+c_{1},
$$

where $a_{1}$ is a constant of $q$-integration. Taking $q$-integral to (10) for $t \in[0, T]$, we obtain

$$
u(t)=\int_{0}^{t}\left(\int_{0}^{\nu} h(s) d_{q} s\right) d_{q} \nu+c_{1} t+c_{2}
$$


Changing the order of $q$-integration, we get

$$
u(t)=\int_{0}^{t}\left(\int_{q s}^{t} h(s) d_{q} \nu\right) d_{q} s+c_{1} t+c_{2}
$$

Therefore, (12) can be written as

$$
u(t)=\int_{0}^{t}(t-q s) h(s) d_{q} s+c_{1} t+c_{2} .
$$

It follows that $c_{2}=u(0)$. Taking the $p_{i}$-integral of (13) from 0 to $\eta$, we get

$$
\begin{aligned}
\int_{0}^{\eta} u(s) d_{p_{i}} s & =\int_{0}^{\eta}\left(\int_{0}^{s}(s-q \nu) h(\nu) d_{q} \nu+c_{1} s+c_{2}\right) d_{p_{i}} s \\
& =\int_{0}^{\eta}\left(\int_{0}^{s}(s-q \nu) h(\nu) d_{q} \nu\right) d_{p_{i}} s+c_{1} \int_{0}^{\eta} s d_{p_{i}} s+c_{2} \eta \\
& =\int_{0}^{\eta}\left(\int_{0}^{s}(s-q \nu) h(\nu) d_{q} \nu\right) d_{p_{i}} s+c_{1} \frac{\eta^{2}}{1+p_{i}}+c_{2} \eta
\end{aligned}
$$

The first condition of (7) implies that

$$
-c_{1} \eta^{2} \sum_{i=1}^{n} \frac{\alpha_{i}}{1+p_{i}}+c_{2}\left(1-\eta \sum_{i=1}^{n} \alpha_{i}\right)=\sum_{i=1}^{n} \alpha_{i} \int_{0}^{\eta}\left(\int_{0}^{s}(s-q \nu) h(\nu) d_{q} \nu\right) d_{p_{i}} s
$$

Form (13), we obtain

$$
u(T)=\int_{0}^{T}(T-q s) h(s) d_{q} s+c_{1} T+c_{2} .
$$

Then, $r_{j}$-integrating of (13) gives

$$
\int_{0}^{\eta} u(s) d_{r_{j}} s=\int_{0}^{\eta}\left(\int_{0}^{s}(s-q \nu) h(\nu) d_{q} \nu\right) d_{r_{j}} s+c_{1} \frac{\eta^{2}}{1+r_{j}}+c_{2} \eta .
$$

The second condition of (7) implies that

$$
\begin{aligned}
& c_{1}\left(T-\eta^{2} \sum_{j=1}^{m} \frac{\beta_{j}}{1+r_{j}}\right)-c_{2}\left(1-\eta \sum_{j=1}^{m} \beta_{j}\right) \\
& =\sum_{j=1}^{m} \beta_{j} \int_{0}^{\eta}\left(\int_{0}^{s}(s-q \nu) h(\nu) d_{q} \nu\right) d_{r_{j}} s-\int_{0}^{T}(T-q s) h(s) d_{q} s .
\end{aligned}
$$


Solving the system of linear equations on the unknown constants $c_{1}$ and $c_{2}$, we obtain

$$
\begin{aligned}
c_{1}= & \left(1-\eta \sum_{j=1}^{m} \beta_{j}\right) \sum_{i=1}^{n} \frac{\alpha_{i}}{\Phi} \int_{0}^{\eta}\left(\int_{0}^{s}(s-q \nu) h(\nu) d_{q} \nu\right) d_{p_{i}} s \\
& -\left(1-\eta \sum_{i=1}^{n} \alpha_{i}\right) \sum_{j=1}^{m} \frac{\beta_{j}}{\Phi} \int_{0}^{\eta}\left(\int_{0}^{s}(s-q \nu) h(\nu) d_{q} \nu\right) d_{r_{j}} s \\
& +\left(1-\eta \sum_{i=1}^{n} \alpha_{i}\right) \int_{0}^{T} \frac{(T-q s)}{\Phi} h(s) d_{q} s, \\
c_{2}= & -\left(T-\eta^{2} \sum_{j=1}^{m} \frac{\beta_{j}}{1+r_{j}}\right) \sum_{j=1}^{m} \frac{\alpha_{i}}{\Phi} \int_{0}^{\eta}\left(\int_{0}^{s}(s-q \nu) h(\nu) d_{q} \nu\right) d_{p_{i}} s \\
& -\left(\eta^{2} \sum_{i=1}^{n} \frac{\alpha_{i}}{1+p_{i}}\right) \sum_{j=1}^{m} \frac{\beta_{j}}{\Phi} \int_{0}^{\eta}\left(\int_{0}^{s}(s-q \nu) h(\nu) d_{q} \nu\right) d_{r_{j}} s \\
& +\eta^{2} \sum_{i=1}^{n} \frac{\alpha_{i}}{1+p_{i}} \int_{0}^{T} \frac{(T-q s)}{\Phi} h(s) d_{q} s .
\end{aligned}
$$

Substituting values of $c_{1}$ and $c_{2}$ in (13), we obtain the solution (8).

For the forthcoming analysis, let $\mathcal{C}=C([0, T], R)$ denote the Banach space of all continuous functions from $[0, T]$ to $R$ endowed with the norm defined by $\|u\|=\sup \{|u(t)|, t \in[0, T]\}$. In view of Lemma 1 and (9), we consider the operator $F: \mathcal{C} \rightarrow \mathcal{C}$ defined by

$$
\begin{aligned}
(F u)(t)= & \int_{0}^{t}(t-q s) f(s, u(s)) d_{q} s \\
& +\left[\left(1-\eta \sum_{i=1}^{n} \alpha_{i}\right) t+\eta^{2} \sum_{i=1}^{n} \frac{\alpha_{i}}{1+p_{i}}\right] \int_{0}^{T} \frac{(T-q s)}{\Phi} f(s, u(s)) d_{q} s \\
& -\left[\left(1-\eta \sum_{i=1}^{n} \alpha_{i}\right) t+\eta^{2} \sum_{i=1}^{n} \frac{\alpha_{i}}{1+p_{i}}\right] \\
& \times \sum_{j=1}^{m} \frac{\beta_{j}}{\Phi} \int_{0}^{\eta} \int_{0}^{s}(s-q \nu) f(\nu, u(\nu)) d_{q} \nu d_{r_{j}} s \\
& +\left[\left(1-\eta \sum_{j=1}^{m} \beta_{j}\right) t-T+\eta^{2} \sum_{j=1}^{m} \frac{\beta_{j}}{1+r_{j}}\right]
\end{aligned}
$$




$$
\times \sum_{i=1}^{n} \frac{\alpha_{i}}{\Phi} \int_{0}^{\eta} \int_{0}^{s}(s-q \nu) f(\nu, u(\nu)) d_{q} \nu d_{p_{i}} s .
$$

Note that the problem (1)-(2) has solutions if and only if the operator equation $F x=x$ has fixed points.

In the following, for the sake of convenience, we set

$$
\begin{aligned}
\Omega= & T^{2}+\left(\left(1+\eta \sum_{i=1}^{n}\left|\alpha_{i}\right|\right) T+\eta^{2} \sum_{i=1}^{n} \frac{\left|\alpha_{i}\right|}{1+p_{i}}\right) \frac{T^{2}}{|\Phi|} \\
& +\left(\left(1+\eta \sum_{i=1}^{n}\left|\alpha_{i}\right|\right) T+\eta^{2} \sum_{i=1}^{n} \frac{\left|\alpha_{i}\right|}{1+p_{i}}\right) \sum_{j=1}^{m} \frac{\left|\beta_{j}\right| \eta^{3}}{|\Phi|\left(r_{j}^{2}+r_{j}+1\right)} \\
& +\left(\eta^{2} \sum_{j=1}^{m} \frac{\left|\beta_{j}\right|}{1+r_{j}}+\eta \sum_{j=1}^{m}\left|\beta_{j}\right|\right) \sum_{i=1}^{n} \frac{\left|\alpha_{i}\right| \eta^{3}}{|\Phi|\left(p_{i}^{2}+p_{i}+1\right)}
\end{aligned}
$$

where $\Phi$ is defined by (9).

\section{Main Results}

Now we are in position to establish the main results. Our first result is based on Banach's fixed point theorem.

Theorem 2. Let $f:[0, T] \times R \rightarrow R$ be a jointly continuous function. In addition, suppose that there exists a positive constant $L$ such that:

$\left(H_{1}\right)|f(t, u(t))-f(t, v(t))| \leq L|u-v|$, for all $t \in[0, T], u, v \in R$.

$\left(H_{2}\right) \Lambda:=L \Omega<1$, where $\Omega$ is defined by (15).

Then the boundary value problem (1)-(2) has a unique solution.

Proof. Assume that $\sup _{t \in[0, T]}|f(t, 0)|=M$, and define a constant $r$ as

$$
r \geq \frac{M \Omega}{1-\delta}
$$

Now, we shall show that $F B_{r} \subset B_{r}$, where $B_{r}=\{u \in \mathcal{C}:\|u\| \leq r\}$. For any $u \in B_{r}$, we get

$$
\|F u\|=\sup _{t \in[0, T]} \mid \int_{0}^{t}(t-q s) f(s, u(s)) d_{q} s
$$




$$
\begin{aligned}
& +\left[\left(1-\eta \sum_{i=1}^{n} \alpha_{i}\right) t+\eta^{2} \sum_{i=1}^{n} \frac{\alpha_{i}}{1+p_{i}}\right] \\
& \times \int_{0}^{T} \frac{(T-q s)}{\Phi} f(s, u(s)) d_{q} s \\
& +\left[\left(1-\eta \sum_{i=1}^{n} \alpha_{i}\right) t+\eta^{2} \sum_{i=1}^{n} \frac{\alpha_{i}}{1+p_{i}}\right] \sum_{j=1}^{m} \frac{\beta_{j}}{\Phi} \\
& \times \int_{0}^{\eta} \int_{0}^{s}(s-q \nu) f(\nu, u(\nu)) d_{q} \nu d_{r_{j}} s \\
& +\left[\left(1-\eta \sum_{j=1}^{m} \beta_{j}\right) t-T+\eta^{2} \sum_{j=1}^{m} \frac{\beta_{j}}{1+r_{j}}\right] \sum_{i=1}^{n} \frac{\alpha_{i}}{\Phi} \\
& \times \int_{0}^{\eta} \int_{0}^{s}(s-q \nu) f(\nu, u(\nu)) d_{q} \nu d_{p_{i}} s \\
& \leq \sup _{t \in[0, T]}\left\{\int_{0}^{t}(t-q s)(|f(s, u(s))-f(s, 0)|+|f(s, 0)|) d_{q} s\right. \\
& +\left|\left(1-\eta \sum_{i=1}^{n} \alpha_{i}\right) t+\eta^{2} \sum_{i=1}^{n} \frac{\alpha_{i}}{1+p_{i}}\right| \\
& \times \int_{0}^{T} \frac{(T-q s)}{|\Phi|}(|f(s, u(s))-f(s, 0)|+|f(s, 0)|) d_{q} s \\
& +\left|\left(1-\eta \sum_{i=1}^{n} \alpha_{i}\right) t+\eta^{2} \sum_{i=1}^{n} \frac{\alpha_{i}}{1+p_{i}}\right| \sum_{j=1}^{m} \frac{\left|\beta_{j}\right|}{|\Phi|} \\
& \times \int_{0}^{\eta} \int_{0}^{s}(s-q \nu)(|f(\nu, u(\nu))-f(\nu, 0)|+|f(\nu, 0)|) d_{q} \nu d_{r_{j}} s \\
& +\left|\left(1-\eta \sum_{j=1}^{m} \beta_{j}\right) t-T+\eta^{2} \sum_{j=1}^{m} \frac{\beta_{j}}{1+r_{j}}\right| \sum_{i=1}^{n} \frac{\left|\alpha_{i}\right|}{|\Phi|} \\
& \left.\times \int_{0}^{\eta} \int_{0}^{s}(s-q \nu)(|f(\nu, u(\nu))-f(\nu, 0)|+|f(\nu, 0)|) d_{q} \nu d_{p_{i}} s\right\} \\
& \leq \sup _{t \in[0, T]}\left\{\int_{0}^{t}(t-q s)(L|u(s)|+|f(s, 0)|) d_{q} s\right. \\
& +\left|\left(1-\eta \sum_{i=1}^{n} \alpha_{i}\right) t+\eta^{2} \sum_{i=1}^{n} \frac{\alpha_{i}}{1+p_{i}}\right| \int_{0}^{T} \frac{(T-q s)}{|\Phi|}(L|u(s)|+|f(s, 0)|) d_{q} s
\end{aligned}
$$




$$
\begin{aligned}
& +\left|\left(1-\eta \sum_{i=1}^{n} \alpha_{i}\right) t+\eta^{2} \sum_{i=1}^{n} \frac{\alpha_{i}}{1+p_{i}}\right| \sum_{j=1}^{m} \frac{\left|\beta_{j}\right|}{|\Phi|} \\
& \times \int_{0}^{\eta} \int_{0}^{s}(s-q \nu)(L|u(\nu)|+|f(\nu, 0)|) d_{q} \nu d_{r_{j}} s \\
& +\left|\left(1-\eta \sum_{j=1}^{m} \beta_{j}\right) t-T+\eta^{2} \sum_{j=1}^{m} \frac{\beta_{j}}{1+r_{j}}\right| \sum_{i=1}^{n} \frac{\left|\alpha_{i}\right|}{|\Phi|} \\
& \left.\times \int_{0}^{\eta} \int_{0}^{s}(s-q \nu)(L|u(\nu)|+|f(\nu, 0)|) d_{q} \nu d_{p_{i}} s\right\} \\
& \leq \sup _{t \in[0, T]}\left\{\int_{0}^{t}(t-q s)(L\|u\|+M) d_{q} s\right. \\
& +\left|\left(1-\eta \sum_{i=1}^{n} \alpha_{i}\right) t+\eta^{2} \sum_{i=1}^{n} \frac{\alpha_{i}}{1+p_{i}}\right| \int_{0}^{T} \frac{(T-q s)}{|\Phi|}(L\|u\|+M) d_{q} s \\
& +\left|\left(1-\eta \sum_{i=1}^{n} \alpha_{i}\right) t+\eta^{2} \sum_{i=1}^{n} \frac{\alpha_{i}}{1+p_{i}}\right| \sum_{j=1}^{m} \frac{\left|\beta_{j}\right|}{|\Phi|} \\
& \times \int_{0}^{\eta} \int_{0}^{s}(s-q \nu)(L\|u\|+M) d_{q} \nu d_{r_{j}} s \\
& +\left|\left(1-\eta \sum_{j=1}^{m} \beta_{j}\right) t-T+\eta^{2} \sum_{j=1}^{m} \frac{\beta_{j}}{1+r_{j}}\right| \sum_{i=1}^{n} \frac{\left|\alpha_{i}\right|}{|\Phi|} \\
& \left.\times \int_{0}^{\eta} \int_{0}^{s}(s-q \nu)(L\|u\|+M) d_{q} \nu d_{p_{i}} s\right\} \\
& \leq \sup _{t \in[0, T]}\left\{( L \| u \| + M ) \left[t^{2}+\left|\left(1-\eta \sum_{i=1}^{n} \alpha_{i}\right) t+\eta^{2} \sum_{i=1}^{n} \frac{\alpha_{i}}{1+p_{i}}\right| \frac{T^{2}}{|\Phi|}\right.\right. \\
& +\left|\left(1-\eta \sum_{i=1}^{n} \alpha_{i}\right) t+\eta^{2} \sum_{i=1}^{n} \frac{\alpha_{i}}{1+p_{i}}\right| \sum_{j=1}^{m} \frac{\left|\beta_{j}\right|}{|\Phi|} \frac{\eta^{3}}{r_{j}^{2}+r_{j}+1} \\
& \left.\left.+\left|\left(1-\eta \sum_{j=1}^{m} \beta_{j}\right) t-T+\eta^{2} \sum_{j=1}^{m} \frac{\beta_{j}}{1+r_{j}}\right| \sum_{i=1}^{n} \frac{\left|\alpha_{i}\right|}{|\Phi|} \frac{\eta^{3}}{p_{i}^{2}+p_{i}+1}\right]\right\} \\
& \leq(L r+M)\left[T^{2}+\left(\left(1+\eta \sum_{i=1}^{n}\left|\alpha_{i}\right|\right) T+\eta^{2} \sum_{i=1}^{n} \frac{\left|\alpha_{i}\right|}{1+p_{i}}\right) \frac{T^{2}}{|\Phi|}\right.
\end{aligned}
$$




$$
\begin{aligned}
& +\left(\left(1+\eta \sum_{i=1}^{n}\left|\alpha_{i}\right|\right) T+\eta^{2} \sum_{i=1}^{n} \frac{\left|\alpha_{i}\right|}{1+p_{i}}\right) \sum_{j=1}^{m} \frac{\left|\beta_{j}\right| \eta^{3}}{|\Phi|\left(r_{j}^{2}+r_{j}+1\right)} \\
& \left.+\left(\eta^{2} \sum_{j=1}^{m} \frac{\left|\beta_{j}\right|}{1+r_{j}}+\eta \sum_{j=1}^{m}\left|\beta_{j}\right|\right) \sum_{i=1}^{n} \frac{\left|\alpha_{i}\right| \eta^{3}}{|\Phi|\left(p_{i}^{2}+p_{i}+1\right)}\right] \\
& \leq(L r+M) \Omega \leq r .
\end{aligned}
$$

Therefore, $F B_{r} \subset B_{r}$. Next, we will show that $F$ is contraction. In the following, from $\left(H_{1}\right)$ for any $u, v \in \mathcal{C}$ and for each $t \in[0, T]$, we have

$$
\begin{aligned}
& \|F u-F v\|=\sup _{t \in[0, T]}|(F u)(t)-(F v)(t)| \\
& =\sup _{t \in[0, T]} \mid \int_{0}^{t}(t-q s)(f(s, u(s))-f(s, v(s))) d_{q} s \\
& +\left[\left(1-\eta \sum_{i=1}^{n} \alpha_{i}\right) t+\eta^{2} \sum_{i=1}^{n} \frac{\alpha_{i}}{1+p_{i}}\right] \\
& \quad \times \int_{0}^{T} \frac{(T-q s)}{\Phi}(f(s, u(s))-f(s, v(s))) d_{q} s \\
& +\left[\left(1-\eta \sum_{i=1}^{n} \alpha_{i}\right) t+\eta^{2} \sum_{i=1}^{n} \frac{\alpha_{i}}{1+p_{i}}\right] \sum_{j=1}^{m} \frac{\beta_{j}}{\Phi} \\
& \times \int_{0}^{\eta} \int_{0}^{s}(s-q \nu)(f(\nu, u(\nu))-f(\nu, v(\nu))) d_{q} \nu d_{r_{j}} s \\
& +\left[\left(1-\eta \sum_{j=1}^{m} \beta_{j}\right) t-T+\eta^{2} \sum_{j=1}^{m} \frac{\beta_{j}}{1+r_{j}}\right] \sum_{i=1}^{n} \frac{\alpha_{i}}{\Phi} \\
& \times \int_{0}^{\eta} \int_{0}^{s}(s-q \nu)(f(\nu, u(\nu))-f(\nu, v(\nu))) d_{q} \nu d_{p_{i}} s \mid \\
& \leq \\
& +L\|u-v\| \mid T^{2}+\left(\left(1+\eta \sum_{i=1}^{n}\left|\alpha_{i}\right|\right) T+\eta^{2} \sum_{i=1}^{n} \frac{\left|\alpha_{i}\right|}{1+p_{i}}\right) \frac{T^{2}}{|\Phi|} \\
& +\left(\left(1+\eta \sum_{i=1}^{n}\left|\alpha_{i}\right|\right) T+\eta^{2} \sum_{i=1}^{n} \frac{\left|\alpha_{i}\right|}{1+p_{i}}\right) \sum_{j=1}^{m} \frac{\left|\beta_{j}\right| \eta^{3}}{|\Phi|\left(r_{j}^{2}+r_{j}+1\right)} \\
& \left.+\left(\eta^{2} \sum_{j=1}^{m} \frac{\left|\beta_{j}\right|}{1+r_{j}}+\eta \sum_{j=1}^{m}\left|\beta_{j}\right|\right) \sum_{i=1}^{n} \frac{\left|\alpha_{i}\right| \eta^{3}}{|\Phi|\left(p_{i}^{2}+p_{i}+1\right)}\right] \\
& +
\end{aligned}
$$




$$
=L \Omega\|u-v\| \leq \Lambda\|u-v\|
$$

From $\left(H_{2}\right)$, we have that $F$ is a contraction. Hence, by the Banach's fixed point theorem, we get that $F$ has a fixed point which is a unique solution of the problem (1)-(2). This completes the proof.

Now, we state a result due to Krasnoselskii [33] which is needed to prove the existence of at least one solution of the problem (1)-(2).

Theorem 3. Let $K$ be a bounded closed convex and nonempty subset of a Banach space $X$. Let $A, B$ be the operators such that:

(i) $A x+B y \in K$ whenever $x, y \in K$,

(ii) $A$ is compact and continuous,

(iii) $B$ is a contraction mapping.

Then there exists $z \in K$ such that $z=A z+B z$.

Our second result is based on the following Krasnoselskii's fixed point theorem.

Theorem 4. Assume that $\left(H_{1}\right)$ and $\left(H_{2}\right)$ hold with

$$
|f(t, u)| \leq \mu(t)
$$

for all $(t, u) \in[0, T] \times R$, where $\mu \in L^{1}\left([0, T], R^{+}\right)$. Then the boundary value problem (1)-(2) has at least one solution on $[0, T]$.

Proof. Setting $\max _{t \in[0, T]}|\mu(t)|=\|\mu\|$, and choosing a constant $r$ such that

$$
r \geq\|\mu\| \Omega
$$

where $\Omega$ is given by (15). Consider $B_{r}=\{u \in \mathcal{C}:\|u\| \leq r\}$. In view of Lemma 2.1 we define the operators $G_{1}$ and $G_{2}$ as follows:

$$
\begin{aligned}
\left(G_{1} u\right)(t)= & \int_{0}^{t}(t-q s) f(s, u(s)) d_{q} s \\
\left(G_{2} u\right)(t)= & {\left[\left(1-\eta \sum_{i=1}^{n} \alpha_{i}\right) t+\eta^{2} \sum_{i=1}^{n} \frac{\alpha_{i}}{1+p_{i}}\right] \int_{0}^{T} \frac{(T-q s)}{\Phi} f(s, u(s)) d_{q} s } \\
& -\left[\left(1-\eta \sum_{i=1}^{n} \alpha_{i}\right) t+\eta^{2} \sum_{i=1}^{n} \frac{\alpha_{i}}{1+p_{i}}\right] \\
& \times \sum_{j=1}^{m} \frac{\beta_{j}}{\Phi} \int_{0}^{\eta} \int_{0}^{s}(s-q \nu) f(\nu, u(\nu)) d_{q} \nu d_{r_{j}} s
\end{aligned}
$$




$$
\begin{aligned}
& +\left[\left(1-\eta \sum_{j=1}^{m} \beta_{j}\right) t-T+\eta^{2} \sum_{j=1}^{m} \frac{\beta_{j}}{1+r_{j}}\right] \\
& \times \sum_{i=1}^{n} \frac{\alpha_{i}}{\Phi} \int_{0}^{\eta} \int_{0}^{s}(s-q \nu) f(\nu, u(\nu)) d_{q} \nu d_{p_{i}} s .
\end{aligned}
$$

Now we shall show that $G_{1}+G_{2}$ has a fixed point in $B_{r}$. For $u, v \in B_{r}$, we have by computing directly, that

$$
\begin{aligned}
\left\|G_{1} u+G_{2} v\right\| \leq & \|u\| \int_{0}^{t}(t-q s) d_{q} s \\
& +\left|\left(1-\eta \sum_{i=1}^{n} \alpha_{i}\right) t+\eta^{2} \sum_{i=1}^{n} \frac{\alpha_{i}}{1+p_{i}}\right| \frac{\| u||}{|\Phi|} \int_{0}^{T}(T-q s) d_{q} s \\
& +\left|\left(1-\eta \sum_{i=1}^{n} \alpha_{i}\right) t+\eta^{2} \sum_{i=1}^{n} \frac{\alpha_{i}}{1+p_{i}}\right| \sum_{j=1}^{m} \frac{\left|\beta_{j}\right|}{|\Phi|} \\
& \times\|u\| \int_{0}^{\eta} \int_{0}^{s}(s-q \nu) d_{q} \nu d_{r_{j}} s \\
& +\left|\left(1-\eta \sum_{j=1}^{m} \beta_{j}\right) t-T+\eta^{2} \sum_{j=1}^{m} \frac{\beta_{j}}{1+r_{j}}\right| \sum_{i=1}^{n} \frac{\left|\alpha_{i}\right|}{|\Phi|} \\
& \times\|u\| \int_{0}^{\eta} \int_{0}^{s}(s-q \nu) d_{q} \nu d_{p_{i}} s \\
\leq & \|u\|\left[T^{2}+\left(\left(1+\eta \sum_{i=1}^{n}\left|\alpha_{i}\right|\right) T+\eta^{2} \sum_{i=1}^{n} \frac{\left|\alpha_{i}\right|}{1+p_{i}}\right) \frac{T^{2}}{|\Phi|}\right. \\
& +\left(\left(1+\eta \sum_{i=1}^{n}\left|\alpha_{i}\right|\right) T+\eta^{2} \sum_{i=1}^{n} \frac{\left|\alpha_{i}\right|}{1+p_{i}}\right) \sum_{j=1}^{m} \frac{\left|\beta_{j}\right| \eta^{3}}{|\Phi|\left(r_{j}^{2}+r_{j}+1\right)} \\
& \left.+\left(\eta^{2} \sum_{j=1}^{m} \frac{\left|\beta_{j}\right|}{1+r_{j}}+\eta \sum_{j=1}^{m}\left|\beta_{j}\right|\right) \sum_{i=1}^{n} \frac{\left|\alpha_{i}\right| \eta^{3}}{|\Phi|\left(p_{i}^{2}+p_{i}+1\right)}\right] \\
\leq & \| u|| \Omega \leq r .
\end{aligned}
$$

Therefore, $G_{1} u+G_{2} v \in B_{r}$. From assumption $\left(H_{1}\right)$ and $\left(H_{2}\right)$ for $u, v \in \mathcal{C}$, we get that

$$
\left\|G_{2} u-G_{2} v\right\| \leq \Lambda\|u-v\|_{\mathcal{C}} .
$$

Hence, $G_{2}$ is a contraction mapping. Next, we shall show that $G_{1}$ is compact and continuous. The continuity of $f$ implies that the operator $G_{1}$ is continuous. 
By using condition (17), we have that $G_{1}$ is uniformly bounded on $B_{r}$ since

$$
\left\|G_{1} u\right\| \leq\|\mu\| T^{2} .
$$

Furthermore, in view of $\left(H_{1}\right)$, we define $\sup _{(t, u) \in[0, T] \times B_{r}}|f(t, u)|=f_{\max }<\infty$, and consequently we get that

$$
\begin{aligned}
& \left|\left(G_{1} u\right)\left(t_{2}\right)-\left(G_{1} v\right)\left(t_{1}\right)\right| \\
& \leq \sup _{(t, u) \in[0, T] \times B_{r}}\left|\int_{0}^{t_{2}}\left(t_{2}-q s\right) f(s, u(s)) d_{q} s-\int_{0}^{t_{1}}\left(t_{1}-q s\right) f(s, u(s)) d_{q} s\right| \\
& \leq \int_{0}^{t_{1}}\left(t_{2}-t_{1}\right)|f(s, u(s))| d_{q} s+\int_{t_{1}}^{t_{2}}\left(t_{2}-q s\right)|f(s, u(s))| d_{q} s \\
& \leq f_{\max }\left(\int_{0}^{t_{1}}\left(t_{2}-t_{1}\right) d_{q} s+\int_{t_{1}}^{t_{2}}\left|t_{2}-q s\right| d_{q} s\right) .
\end{aligned}
$$

Actually, as $t_{2} \rightarrow t_{1}$, the right-hand side of the above inequality, which is independent of $u$, tends to zero. Therefore, $G_{1}$ is equicontinuous. Since $G_{1}$ is uniformly bounded and equicontinuous on $B_{r}$, we get that $G_{1}$ is relatively compact on $B_{r}$. Hence, by the Arzelá-Ascoli Theorem, $G_{1}$ is compact on $B_{r}$. Thus, all the assumptions of Theorem 3 are satisfied. By Theorem 4, we have that the boundary value problem (1)-(2) has at least one solution on $[0, T]$. This completes the proof.

Next, we prove the existence of solutions for the problem (1)-(2) by using Leray-Schauder degree theory.

Theorem 5. Let $f:[0, T] \times R \rightarrow R$. Suppose that there exist constants $0 \leq \kappa<\Omega^{-1}$, where $\Omega$ is given by (15) and $M>0$ such that $|f(t, u)| \leq \kappa|u|+M$ for all $t \in[0, T], u \in R$. Then the boundary value problem (1)-(2) has at least one solution on $[0, T]$.

Proof. We define an operator $F: \mathcal{C} \rightarrow \mathcal{C}$ as in (14). In view of the fixed point problem

$$
u=F u \text {. }
$$

We are going to prove the existence of at least one solution $u \in C[0, T]$ satisfying (20). Set a ball $B_{R} \subset C[0, T]$, as

$$
B_{R}=\left\{u \in \mathcal{C}: \max _{t \in[0, T]}|u(t)|<R\right\},
$$

where a constant radius $R>0$. Hence, we will show that $F: \bar{B}_{R} \rightarrow C[0, T]$ satisfies a condition

$$
u \neq \lambda F u, \quad \forall u \in \partial B_{R}, \quad \forall \lambda \in[0,1] .
$$


We set

$$
H(\lambda, u)=\lambda F u, \quad u \in \mathcal{C}, \quad \lambda \in[0,1] .
$$

As shown in Theorem 2 and 4, we have that the operator $F$ that is continuous, uniformly bounded and equicontinuous. Then, by the Arzelá-Ascoli theorem, a continuous map $h_{\lambda}$ defined by $h_{\lambda}(u)=u-H(\lambda, u)=u-\lambda F u$ is completely continuous. If (21) holds, then the following Leray-Schauder degrees are well defined and by the homotopy invariance of topological degree, it follows that

$$
\begin{aligned}
\operatorname{deg}\left(h_{\lambda}, B_{R}, 0\right) & =\operatorname{deg}\left(I-\lambda F, B_{R}, 0\right)=\operatorname{deg}\left(h, b_{R}, 0\right) \\
& =\operatorname{deg}\left(h_{0}, B_{R}, 0\right)=\operatorname{deg}\left(I, B_{R}, 0\right)=1 \neq 0, \quad 0 \in B_{R},
\end{aligned}
$$

where $I$ denotes the unit operator. By the nonzero property of Leray-Schauder degree, $h_{1}(u)=u-F u=0$ for at least one $u \in B_{R}$. Let us assume that $u=\lambda F u$ for some $\lambda \in[0,1]$ and for all $t \in[0, T]$ so that

$$
\begin{aligned}
|u(t)|= & |\lambda(F u)(t)| \\
\leq & \int_{0}^{t}(t-q s)|f(s, u(s))| d_{q} s \\
& +\left|\left(1-\eta \sum_{i=1}^{n} \alpha_{i}\right) t+\eta^{2} \sum_{i=1}^{n} \frac{\alpha_{i}}{1+p_{i}}\right| \int_{0}^{T} \frac{(T-q s)}{|\Phi|}|f(s, u(s))| d_{q} s \\
& +\left|\left(1-\eta \sum_{i=1}^{n} \alpha_{i}\right) t+\eta^{2} \sum_{i=1}^{n} \frac{\alpha_{i}}{1+p_{i}}\right| \\
& \times \sum_{j=1}^{m} \frac{\left|\beta_{j}\right|}{|\Phi|} \int_{0}^{\eta} \int_{0}^{s}(s-q \nu)|f(\nu, u(\nu))| d_{q} \nu d_{r_{j}} s \\
& +\left|\left(1-\eta \sum_{j=1}^{m} \beta_{j}\right) t-T+\eta^{2} \sum_{j=1}^{m} \frac{\beta_{j}}{1+r_{j}}\right| \\
& \times \sum_{i=1}^{n} \frac{\left|\alpha_{i}\right|}{|\Phi|} \int_{0}^{\eta} \int_{0}^{s}(s-q \nu)|f(\nu, u(\nu))| d_{q} \nu d_{p_{i}} s \\
\leq & (\kappa|u|+M) \mid \int_{0}^{t}(t-q s) d_{q} s \\
& +\left|\left(1-\eta \sum_{i=1}^{n} \alpha_{i}\right) t+\eta^{2} \sum_{i=1}^{n} \frac{\alpha_{i}}{1+p_{i}}\right| \int_{0}^{T} \frac{(T-q s)}{|\Phi|} d_{q} s \\
& \left|\left(1-\alpha_{i}\right) t+\eta^{2} \sum_{i=1}^{n} \frac{\alpha_{i}}{1+p_{i}}\right|
\end{aligned}
$$




$$
\begin{aligned}
& \times \sum_{j=1}^{m} \frac{\left|\beta_{j}\right|}{|\Phi|} \int_{0}^{\eta} \int_{0}^{s}(s-q \nu) d_{q} \nu d_{r_{j}} s \\
+ & \left|\left(1-\eta \sum_{j=1}^{m} \beta_{j}\right) t-T+\eta^{2} \sum_{j=1}^{m} \frac{\beta_{j}}{1+r_{j}}\right| \\
& \left.\times \sum_{i=1}^{n} \frac{\left|\alpha_{i}\right|}{|\Phi|} \int_{0}^{\eta} \int_{0}^{s}(s-q \nu) d_{q} \nu d_{p_{i}} s\right] \\
\leq & \frac{(\kappa|u|+M)}{1+q}\left[T^{2}+\left(\left(1+\eta \sum_{i=1}^{n}\left|\alpha_{i}\right|\right) T+\eta^{2} \sum_{i=1}^{n} \frac{\left|\alpha_{i}\right|}{1+p_{i}}\right) \frac{T^{2}}{|\Phi|}\right. \\
+ & \left(\left(1+\eta \sum_{i=1}^{n}\left|\alpha_{i}\right|\right) T+\eta^{2} \sum_{i=1}^{n} \frac{\left|\alpha_{i}\right|}{1+p_{i}}\right) \sum_{j=1}^{m} \frac{\left|\beta_{j}\right| \eta^{3}}{|\Phi|\left(r_{j}^{2}+r_{j}+1\right)} \\
+ & \left.\left(\eta^{2} \sum_{j=1}^{m} \frac{\left|\beta_{j}\right|}{1+r_{j}}+\eta \sum_{j=1}^{m}\left|\beta_{j}\right|\right) \sum_{i=1}^{n} \frac{\left|\alpha_{i}\right| \eta^{3}}{|\Phi|\left(p_{i}^{2}+p_{i}+1\right)}\right] \\
= & (\kappa|u|+M) \Omega,
\end{aligned}
$$

which on taking norm $\sup _{t \in[0, T]}|u(t)|=\|u\|$ and solving for $\|u\|$, yields

$$
\|u\| \leq \frac{M \Omega}{1-\kappa \Omega} .
$$

If $R=\frac{M \Omega}{1-\kappa \Omega}+1$, inequality (21) holds. This completes the proof.

\section{Some Examples}

Example 6. Consider the following three-point multi-term $q$-integral boundary value problem

$$
\begin{gathered}
D_{\frac{1}{2}}^{2} u(t)=\frac{1}{(t+4)^{2}} \cdot \frac{|u|}{|u|+1}, \quad t \in[0, \sqrt{2}] . \\
u(0)=\frac{\sqrt{3}}{2} \int_{0}^{\frac{\sqrt{2}}{2}} u(s) d_{\frac{1}{4}} s-\int_{0}^{\frac{\sqrt{2}}{2}} u(s) d_{\frac{2}{3}} s \\
u(\sqrt{2})=-\sqrt{7} \int_{0}^{\frac{\sqrt{2}}{2}} u(s) d_{\frac{1}{2}} s+\frac{\sqrt{\pi}}{2} \int_{0}^{\frac{\sqrt{2}}{2}} u(s) d_{\frac{3}{4}} s-\frac{3}{2} \int_{0}^{\frac{\sqrt{2}}{2}} u(s) d_{\frac{4}{5}} s .
\end{gathered}
$$


Set $q=1 / 2, T=\sqrt{2}, \eta=\sqrt{2} / 2, n=2, \alpha_{1}=\sqrt{3} / 2, \alpha_{2}=-1, p_{1}=1 / 4, p_{2}=$ $2 / 3, m=3, \beta_{1}=-\sqrt{7}, \beta_{2}=\sqrt{\pi} / 2, \beta_{3}=-3 / 2, r_{1}=1 / 2, r_{2}=3 / 4, r_{3}=4 / 5$, $f(t, u)=\left(1 /(t+4)^{2}\right)(|u| /(1+|u|))$. Since $|f(t, u)-f(t, v)| \leq(1 / 16)|u-v|$, $\left(H_{1}\right)$ is satisfied with $\eta^{2}\left(\sum_{j=1}^{m} \frac{\beta_{j}}{1+r_{j}}-\sum_{i=1}^{n} \frac{\alpha_{i}\left(1-\eta \sum_{j=1}^{m} \beta_{j}\right)}{1+p_{i}}\right)-T \approx-4.595866$,

$$
\begin{aligned}
\Phi= & \left(1-\eta \sum_{i=1}^{n} \alpha_{i}\right)\left(\eta^{2} \sum_{j=1}^{m} \frac{\beta_{j}}{1+r_{j}}-T\right)-\eta^{2} \sum_{i=1}^{n} \frac{\alpha_{i}\left(1-\eta \sum_{j=1}^{m} \beta_{j}\right)}{1+p_{i}} \\
\approx & -2.845975, \\
\Omega= & T^{2}+\left(\left(1+\eta \sum_{i=1}^{n}\left|\alpha_{i}\right|\right) T+\eta^{2} \sum_{i=1}^{n} \frac{\left|\alpha_{i}\right|}{1+p_{i}}\right) \frac{T^{2}}{|\Phi|} \\
& +\left(\left(1+\eta \sum_{i=1}^{n}\left|\alpha_{i}\right|\right) T+\eta^{2} \sum_{i=1}^{n} \frac{\left|\alpha_{i}\right|}{1+p_{i}}\right) \sum_{j=1}^{m} \frac{\left|\beta_{j}\right| \eta^{3}}{|\Phi|\left(r_{j}^{2}+r_{j}+1\right)} \\
& +\left(\eta^{2} \sum_{j=1}^{m} \frac{\left|\beta_{j}\right|}{1+r_{j}}+\eta \sum_{j=1}^{m}\left|\beta_{j}\right|\right) \sum_{i=1}^{n} \frac{\left|\alpha_{i}\right| \eta^{3}}{|\Phi|\left(p_{i}^{2}+p_{i}+1\right)}
\end{aligned}
$$

$\approx 11.093692$,

$L=1 / 16$. Hence $\Lambda:=L \Omega \approx 0.693356<1$. Therefore, by Theorem 2 , the boundary value problem (22)-(23) has a unique solution on $[0,2]$.

Example 7. Consider the following three-point multi-term $q$-integral boundary value problem

$$
\begin{gathered}
D_{\frac{2}{3}}^{2} u(t)=\frac{\sin (4 \pi u)}{16 \pi}+\frac{|u|}{|u|+1}, \quad t \in[0,1] . \\
u(0)=\sqrt{3} \int_{0}^{\frac{1}{2}} u(s) d_{\frac{1}{2}} s-\frac{7}{2} \int_{0}^{\frac{1}{2}} u(s) d_{\frac{2}{3}} s-5 \int_{0}^{\frac{1}{2}} u(s) d_{\frac{3}{4}} s \\
u(1)=\frac{1}{4} \int_{0}^{\frac{1}{2}} u(s) d_{\frac{1}{4}} s-\frac{1}{5} \int_{0}^{\frac{1}{2}} u(s) d_{\frac{1}{3}} s-\frac{1}{2} \int_{0}^{\frac{1}{2}} u(s) d_{\frac{1}{5}} s .
\end{gathered}
$$

Set $q=2 / 3, T=1, \eta=1 / 2, n=3, \alpha_{1}=\sqrt{3}, \alpha_{2}=-7 / 2, \alpha_{3}=-5, p_{1}=1 / 2$, $p_{2}=2 / 3, p_{3}=3 / 4, m=3, \beta_{1}=-1 / 5, \beta_{2}=1 / 4, \beta_{3}=-1 / 2, r_{1}=1 / 3$, $r_{2}=1 / 4, r_{3}=1 / 5$, Here, $|f(t, u)|=|\sin (4 \pi u) / 16 \pi+| u|/(1+|u|)| \leq(|u| / 4)+1$. So, $M=1, \eta^{2}\left(\sum_{j=1}^{m} \frac{\beta_{j}}{1+r_{j}}-\sum_{i=1}^{n} \frac{\alpha_{i}\left(1-\eta \sum_{j=1}^{m} \beta_{j}\right)}{1+p_{i}}\right)-T \approx 0.072831$, and

$$
\Phi=\left(1-\eta \sum_{i=1}^{n} \alpha_{i}\right)\left(\eta^{2} \sum_{j=1}^{m} \frac{\beta_{j}}{1+r_{j}}-T\right)-\eta^{2} \sum_{i=1}^{n} \frac{\alpha_{i}\left(1-\eta \sum_{j=1}^{m} \beta_{j}\right)}{1+p_{i}}
$$




$$
\begin{aligned}
\approx & -3.621341, \\
\Omega= & T^{2}+\left(\left(1+\eta \sum_{i=1}^{n}\left|\alpha_{i}\right|\right) T+\eta^{2} \sum_{i=1}^{n} \frac{\left|\alpha_{i}\right|}{1+p_{i}}\right) \frac{T^{2}}{|\Phi|} \\
& +\left(\left(1+\eta \sum_{i=1}^{n}\left|\alpha_{i}\right|\right) T+\eta^{2} \sum_{i=1}^{n} \frac{\left|\alpha_{i}\right|}{1+p_{i}}\right) \sum_{j=1}^{m} \frac{\left|\beta_{j}\right| \eta^{3}}{|\Phi|\left(r_{j}^{2}+r_{j}+1\right)} \\
& +\left(\eta^{2} \sum_{j=1}^{m} \frac{\left|\beta_{j}\right|}{1+r_{j}}+\eta \sum_{j=1}^{m}\left|\beta_{j}\right|\right) \sum_{i=1}^{n} \frac{\left|\alpha_{i}\right| \eta^{3}}{|\Phi|\left(p_{i}^{2}+p_{i}+1\right)} \\
\approx & 3.970666, \\
\kappa= & \frac{1}{4}<\frac{1}{\Omega} \approx 0.251847 .
\end{aligned}
$$

Hence, by Theorem 5, the boundary value problem (24)-(25) has at least one solution on $[0,1]$.

\section{Acknowledgments}

This research was funded by Faculty of Applied Science, King Mongkut's University of Technology North Bangkok, Thailand. Contract No. 5642108.

\section{References}

[1] F.H. Jackson, On q-difference equations, American J. Math., 32, no. 4 (1910), 305-314.

[2] R.D. Carmichael, The general theory of linear $q$-difference equations, American J. Math., 34, no. 2 (1912), 147-168.

[3] T.E. Mason, On properties of the solutions of linear $q$-difference equations with entire function coefficients, American J. Math., 37, no. 4 (1915), 439-444.

[4] C.R. Adams, On the linear ordinary q-difference equation, American Math. Ser. II, 30 (1929), 195-205.

[5] W.J. Trjitzinsky, Analytic theory of linear $q$-differece equations, Acta Mathematica, 61, no. 6 (1933), 1-38. 
[6] T. Ernst, A new notation for q-calculus and a new q-Taylor formula, U.U.D.M. Report, Department of Mathematics, Uppsala University, 1999.

[7] R.J. Finkelstein, q-Field theory, Lett. Math. Phys., 34, no. 2 (1995), 169176.

[8] R.J. Finkelstein, q-deformation of the Lorentz group, J. Math. Phys., 37, no. 2 (1996), 953-964.

[9] R. Floreanini, L. Vinet, Automorphisms of the $q$-oscillator algebra and basic orthogonal polynomials, Phys. Lett. A, 180, no. 6 (1993), 393-401.

[10] R. Floreanini, L. Vinet, Symmetries of the $q$-difference heat equation, Lett. Math. Phys., 32, no. 1 (1994), 37-44.

[11] R. Floreanini, L. Vinet, $q$-gamma and $q$-beta functions in quantum algebra representation theory, J. Comput. Appl. Math., 68, no.1-2 (1996), 57-68.

[12] P.G.O. Freund, A.V. Zabrodin, The spectral problem for the $q$-KnizhnikZamolodchikov equation and continuous $q$-Jacobi polynomials, Comm. Math. Phys., 173, no. 1 (1995), 17-42.

[13] G. Gasper, M. Rahman, Basic Hypergeometric Series, Cambridge University Press, Cambridge, UK, 1990.

[14] G.N. Han, J. Zeng, On a $q$-sequence that generalizes the median Genocchi numbers, Ann. Sci. Math. Quebec, 23, no. 1 (1999), 63-72.

[15] V. Kac, P. Cheung, Quantum Calculus, Springer, New York, NY, USA, 2002.

[16] G. Bangerezako, Variational q-calculus, J. Math. Anal. Appl., 289, no. 2 (2004), 650-665.

[17] G. Bangerezako, Variational calculus on $q$-nonuniform lattices, J. Math. Anal. Appl., 306 (2005), 161-179.

[18] M. Bochner, M. Unal, Kneser theorem in quantum calculus, J. Phys. A: Math. Gen., 38 (2005), 6729-6739.

[19] M. Bohner, G.Sh. Guseinov, The $h$-Laplace and $q$-Laplace transforms, $J$. Math. Anal. Appl., 365 (2010), 75-92. 
[20] A. Dobrogowska, A. Odzijewicz, Second order $q$-difference equations solvable by factorization method, J. Comput. Appl. Math., 193, no. 1 (2006), 319-346.

[21] G. Gasper, M. Rahman, Some systems of multivariable orthogonal $q$-Racah polynomials, Ramanujan J., 13, no. 1-3 (2007), 389-405.

[22] M.E.H. Ismail, P. Simeonov, $q$-difference operators for orthogonal polynomials, J. Comput. Appl. Math., 233, no. 3 (2009), 749-761.

[23] M. El-Shahed, H.A. Hassan, Positive solutions of $q$-difference equation, Proc. Amer. Math. Soc., 138, no. 5 (2010), 1733-1738.

[24] T. Ernst, A Comprehensive Treatment of q-Calculus, Birkhäuser, New York, 2012.

[25] G.C. Wu, Variational iteration method for $q$-difference equations of second order, J. Appl. Math., 2012, ID 102850 (2012), 5 pages.

[26] J. Zhang, G. Wang, J. Chen, R. Zhao, Some results on q-difference equations, Adv. Diff. Equ., 2012, article 191, 2012

[27] B. Ahmad, S.K. Ntouyas, I.K. Purnaras, Existence results for nonlinear $q$-difference equations with nonlocal boundary conditions, Commun. Appl. Nonlinear Anal., 19 (2012), 59-72.

[28] B. Ahmad, J.J. Nieto, On nonlocal boundary value problems of nonlinear q-difference equations, Adv. Diff. Equ., 2012, article 81, 2012.

[29] B. Ahmad, S.K. Ntouyas, Boundary value problems for $q$-difference inclusions, Abstract Appl. Anal., 2011, ID 292860 (2011), 15 pages.

[30] B. Ahmad, Boundary-value problems for nonlinear third-order $q$-difference equations, Electron. J. Diff. Equ., 94 (2011), 1-7.

[31] B. Ahmad, A. Alsaedi, S.K. Ntouyas, A study of second-order $q$-difference equations with boundary conditions, Adv. Diff. Equ., 2012, article 35, 2012.

[32] N. Pongarm, S. Asawasamrit, J. Tariboon, Sequential derivatives of nonlinear $q$-difference equations with three-point $q$-integral boundary conditions, J. Appl. Math., 2013, ID 605169 (2013), 9 pages. 
[33] M.A. Krasnoselskii, Two remarks on the method of successive approximations, Uspekhi Mate. Nauk., 10 (1955), 123-127. 